\title{
How effective are social norms interventions in changing the clinical behaviours of healthcare workers? A systematic review and meta-analysis
}

Mei Yee Tang ${ }^{1,2^{*}}$ (D) Sarah Rhodes ${ }^{1}$, Rachael Powell ${ }^{3}$, Laura McGowan ${ }^{3}$, Elizabeth Howarth', Benjamin Brown ${ }^{4,5}$ and Sarah Cotterill ${ }^{1}$

\begin{abstract}
Background: Healthcare workers perform clinical behaviours which impact on patient diagnoses, care, treatment and recovery. Some methods of supporting healthcare workers in changing their behaviour make use of social norms by exposing healthcare workers to the beliefs, values, attitudes or behaviours of a reference group or person. This review aimed to evaluate evidence on (i) the effect of social norms interventions on healthcare worker clinical behaviour change and (ii) the contexts, modes of delivery and behaviour change techniques (BCTs) associated with effectiveness.
\end{abstract}

Methods: Systematic review and meta-analysis of randomised controlled trials. Searches were undertaken in seven databases. The primary outcome was compliance with a desired healthcare worker clinical behaviour and the secondary outcome was patient health outcomes. Outcomes were converted into standardised mean differences (SMDs). We performed meta-analyses and presented forest plots, stratified by five social norms BCTs (social comparison, credible source, social reward, social incentive and information about others' approval). Sources of variation in social norms BCTs, context and mode of delivery were explored using forest plots, meta-regression and network meta-analysis.

(Continued on next page)

\footnotetext{
* Correspondence: meiyee.tang@newcastle.ac.uk

${ }^{1}$ Centre for Biostatistics, Division of Population Health, Health Services

Research and Primary Care, School of Health Sciences, Faculty of Biology Medicine and Health, University of Manchester, Oxford Road, Manchester M13 9PL, UK

${ }^{2}$ National Institute of Health Research Behavioural Science Policy Research Unit, Population Health Sciences, Baddiley-Clark Building, Faculty of Medical Sciences, Newcastle University, Newcastle Upon Tyne NE2 4AX, UK

Full list of author information is available at the end of the article
}

(c) The Author(s). 2021 Open Access This article is licensed under a Creative Commons Attribution 4.0 International License, which permits use, sharing, adaptation, distribution and reproduction in any medium or format, as long as you give appropriate credit to the original author(s) and the source, provide a link to the Creative Commons licence, and indicate if changes were made. The images or other third party material in this article are included in the article's Creative Commons licence, unless indicated otherwise in a credit line to the material. If material is not included in the article's Creative Commons licence and your intended use is not permitted by statutory regulation or exceeds the permitted use, you will need to obtain permission directly from the copyright holder. To view a copy of this licence, visit http://creativecommons.org/licenses/by/4.0/. The Creative Commons Public Domain Dedication waiver (http://creativecommons.org/publicdomain/zero/1.0/) applies to the data made available in this article, unless otherwise stated in a credit line to the data. 
(Continued from previous page)

Results: Combined data from 116 trials suggested that social norms interventions were associated with an improvement in healthcare worker clinical behaviour outcomes of 0.08 SMDs $(95 \% \mathrm{Cl} 0.07$ to 0.10$)(n=100$ comparisons), and an improvement in patient health outcomes of 0.17 SMDs (95\% Cl 0.14 to 0.20$)(n=14)$, on average. Heterogeneity was high, with an overall $P^{2}$ of $85.4 \%$ (healthcare worker clinical behaviour) and $91.5 \%$ (patient health outcomes). Credible source was more effective on average, compared to control conditions (SMD $0.30,95 \% \mathrm{Cl} 0.13$ to $0.47, n=7)$. Social comparison also appeared effective, both on its own (SMD 0.05, 95\%Cl 0.03 to $0.08, n=33)$ and with other BCTs, and seemed particularly effective when combined with prompts/cues $(0.33,95 \% \mathrm{Cl}$ 0.22 to $0.44, n=5)$.

Conclusions: Social norms interventions appeared to be an effective method of changing the clinical behaviour of healthcare workers and have a positive effect on patient health outcomes in a variety of health service contexts. Although the overall result is modest and variable, there is the potential for social norms interventions to be applied at large scale.

Trial registration: PROSPERO CRD42016045718.

Keywords: Systematic review, Meta-analysis, Health professional behaviour, Social norm, Social comparison, Information about others' approval, Credible source, Social reward, Social incentive, Audit and feedback

\section{Contributions to the literature}

- This is the first systematic review and meta-analysis on the use of social norms interventions to change the clinical behaviour of healthcare workers, and the results suggest that, on average, these interventions are effective.

- Social norms interventions may be effective across a range of health service contexts and modes of delivery, but the effects are variable.

- These findings contribute to a recognised gap in the literature, by highlighting which social norms interventions may be most effective: this can inform the design of future interventions aimed at improving health professional practice.

\section{Background}

Healthcare workers routinely perform behaviours in clinical settings which impact all aspects of patient care including diagnoses, treatment and recovery. There are best-practice guidelines for many of these clinical behaviours. For example, regular blood glucose testing for diabetic patients. Healthcare workers face many challenges in following evidence-based professional practice such as lack of time, competing demands and requests from patients. Although there are no reliable published estimates of how well healthcare workers follow best clinical practices, 1 in 20 hospital admissions is caused by adverse drug events [1], and approximately half of these globally are believed to be due to lapses in best practice in terms of prescribing or monitoring behaviours by clinicians [2].

Social influences are important in clinical practice: prescribers of antibiotics have reported that pressure from patients and other prescribers in their networks influence their prescribing behaviours [3]. Social norms can be broadly considered as the perceived implicit or explicit behavioural rules that one uses to determine the appropriate and/or typical expectations, beliefs, attitudes and behaviours of a social reference person or group [4]. We have defined a social norms intervention as one which seeks to change the clinical behaviour of a target healthcare worker by exposing them to the values, beliefs, attitudes or behaviours of a reference group or person. The target healthcare worker is the person at whom a social norms intervention is aimed, with a view to changing their clinical behaviour. The reference person or group describes a person or group whose values, beliefs or behaviours are exposed to the target. Social norms interventions sometimes report a peer benchmark, such as the top $10 \%$ of the reference group or the average performance: the downside of the average approach is that the above-average performers will receive feedback suggesting that they are already performing better than their peers, and this may lead them to reduce their effort [5].

Behaviour change interventions based on social norms may help overcome barriers to healthcare workers implementing recommended practice through: persuasion, encouraging collaboration to achieve change, observing good practice from elsewhere and support from management [6]. There are various explanations of the processes through which social norms impact on behaviour according to social and health psychology theories. Social comparison theory [7] proposes that individuals draw on social comparisons to evaluate one's abilities and perform behaviours which will bring one's abilities in line with those of others in the group. According to the social identity perspective [8], people make 
evaluations about their own group ('in group norms') against other groups ('out group norms'). They are motivated to preserve their social identity (as part of their 'in group') by behaving in similar ways to the group's normative behaviour. 'Subjective norm' is a construct within the Theory of Planned Behaviour [9], which describes an individual's perception of whether valued others think they should perform a behaviour, combined with a motivation to comply with others' beliefs.

A social norms intervention with a descriptive norms [10] message provides the target with information about the behaviour of others in the reference group (such as providing a nurse with information about the behaviours of nurses regarding wound dressing). An injunctive norms message provides the target worker with information about the values, beliefs or attitudes of the reference group towards a particular behaviour, conveying social approval or disapproval (e.g. saying that colleagues disapprove of ordering unnecessary tests). This includes approval, praise, commendation, applause or thanks.

Audit and feedback (A\&F) is a quality improvement technique used by health services, where data is collected on healthcare worker performance and then a summary is reported back to the individual [11]. Social norms interventions are sometimes included as one component of A\&F, usually by providing descriptive norms of others' behaviour [12, 13]. A\&F has already been shown to be effective in changing healthcare worker behaviour, but with large variation in outcomes depending on the context and the intervention design [14]. There is a need to understand the ingredients for successful A\&F [11, 15], and the effects or mechanisms of the 'social influence' constituents of A\&F have been identified as topics for further research [11]. Our review contributes to this important research agenda by systematically examining the evidence for using social norms interventions with healthcare workers.

Identification of the individual components within social norms interventions can aid understanding of the precise aspects that influence behaviour. The Behaviour Change Techniques Taxonomy v1 (BCTTv1) [16] is a framework for classifying BCTs, which are the 'active ingredients' of behaviour change interventions. The taxonomy defines 93 distinct BCTs, grouped into categories. There is no explicit category that relates to social norms. For this review, five BCTs were considered to involve social norms: '6.2. Social Comparison', '6.3. Information about Others' Approval', '9.1. Credible Source', '10.4. Social Reward', and '10.5. Social Incentive'. The numbers follow the BCTTv1 labelling and definitions are listed in Table 1.

The aim was to conduct a systematic review to assess the impact on healthcare workers' compliance with professional practice recommendations of interventions delivering social norms BCTs, compared to controls. Two research questions were addressed:

1. What is the effect of interventions containing social norms BCTs on (a) the clinical behaviour of healthcare workers, and (b) resulting patient health outcomes?

2. Which contexts, modes of delivery and behaviour change techniques are associated with the effectiveness of social norms interventions on healthcare worker clinical behaviour change?

\section{Methods}

The study design was a systematic review with metaanalysis [18], meta-regression [19] and network metaanalysis [20]. This paper follows the Preferred Reporting Items for Systematic Reviews and Meta-Analyses (PRIS MA) statement [21]. Six members of the public attended workshops to discuss the relevance of the review to patients and carers, study design and dissemination. The group felt that patients can potentially have a role in changing healthcare worker behaviour, for example by reminding healthcare workers to wash their hands; or telling the General Practitioner (GP) they do not want antibiotics for a cold, although they were cynical about whether doctors would listen. In response, we changed our data collection to record whether any studies considered patients' role in social norms interventions. Their advice on how to interpret our results to a broad audience will influence our future dissemination plans. An independent study steering committee, including a member of the public, provided encouragement and counsel throughout the project.

\section{Protocol and registration}

The study was registered on PROSPERO (CRD42016045718) and a protocol is available [17].

\section{Searches}

A search strategy was developed, following an iterative process of scoping searches. In July 2018, searches were undertaken in MEDLINE, PsycINFO, EMBASE, CINA HL, BNI, Cochrane CENTRAL and Web of Science (see Appendix 1). Backward and forward citation searching was not conducted, as per the protocol, due to time constraints.

\section{Study inclusion criteria}

Studies were included if they met the criteria in Table 2.

\section{Screening}

Covidence was used to facilitate screening and data extraction [22]. One reviewer screened all titles and abstracts against the inclusion criteria; a second reviewer 


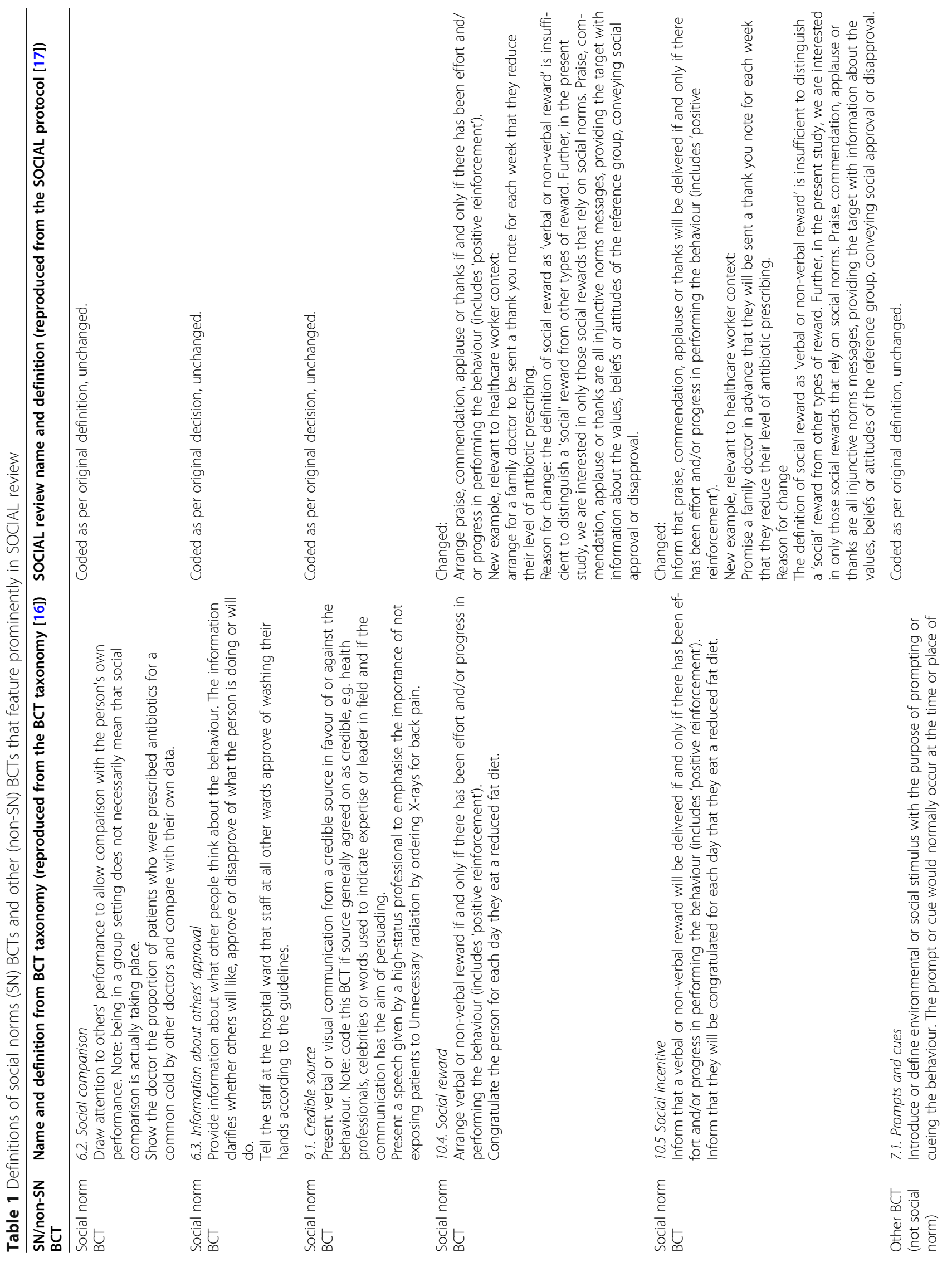




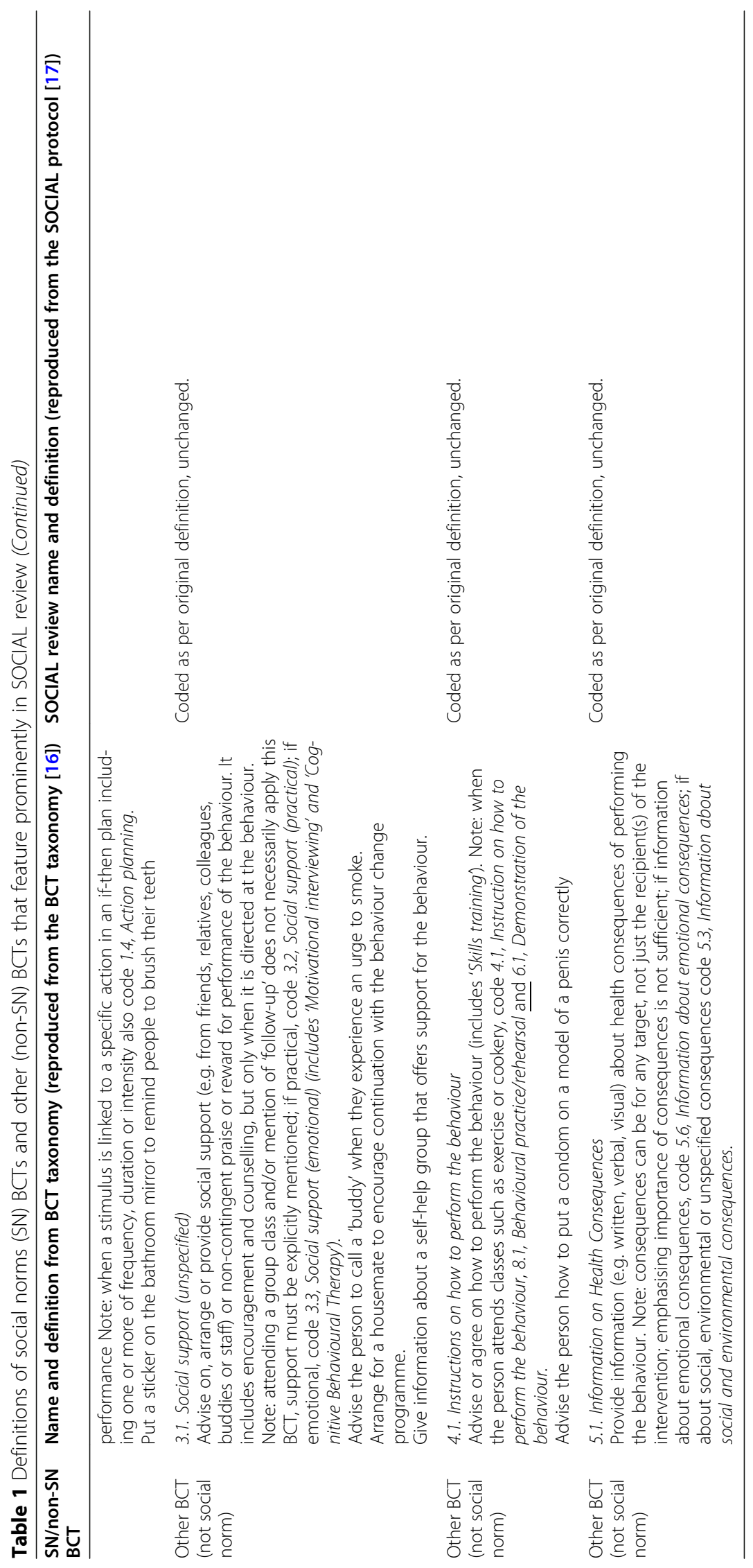


Table 2 Inclusion criteria

\begin{tabular}{ll}
\hline PICOS criterion & Description \\
\hline Population & Healthcare workers, including managers and those in training. \\
Intervention & $\begin{array}{l}\text { A social norms intervention in a (non-simulated) healthcare setting that seeks to change the clinical behaviour } \\
\text { of target population by exposing them to the values, beliefs, attitudes, or behaviours of a reference group or } \\
\text { person. }\end{array}$ \\
Comparison/control & $\begin{array}{l}\text { No restrictions on the comparators. } \\
\text { Outcomes }\end{array}$ \\
Primary outcome of interest was compliance with the desired clinical behaviour. Secondary outcomes were \\
patient health-related outcomes. \\
Randomised controlled trials published in peer-reviewed journals, in English Language. Grey literature was not \\
eligible for inclusion.
\end{tabular}

screened a $20 \%$ random sample to assess reliability. Studies included to the full-text stage were independently screened by two researchers. Any disagreements were resolved through discussion, moderation of a third researcher or team review.

\section{Data extraction}

Data from included studies were extracted using a tailored data extraction form (Appendix 2) [23]. Information relating to the population and setting, methods, participant characteristics, intervention characteristics (delivery and BCT content), comparators, outcomes and results were extracted.

For the primary outcome (healthcare worker clinical behaviour), we extracted all available summary data on compliance of the healthcare worker with the desired behaviour at the time point closest to 6 months post-randomisation. Where multiple measures of compliance were reported we followed this list of priorities: (a) reported in sufficient detail to calculate standardised mean difference, (b) observed rather than self-report, (c) appropriate adjustment for clustering, (d) continuous measure, (e) final score rather than change from baseline, (f) described as primary outcome, (g) used to calculate sample size and (h) reported first. A similar approach was followed for patient health outcomes.

All identified BCTs (including both social norms and non-social norms) in all control and intervention arms of included studies were independently coded by two trained researchers using the BCTTv1 [16] and recorded on a BCT extraction form (Appendix 3). The intervention descriptions from all relevant papers (including protocols, process evaluations or additional sources cited in the included studies) were coded to capture the BCTs as closely as possible. Inter-rater reliability for each of the $\mathrm{BCT}$ s that were present at least once across all arms was assessed using the prevalence and bias-adjusted kappa (PABAK) statistic (see Appendix 4), which adjusts for both the prevalence and occurrence of BCTs [24]. In circumstances where prevalence is low, the widely used chance-corrected kappa statistic is likely to underestimate reliability as it is highly dependent on prevalence [25].

\section{Study quality assessment}

Risk of bias was independently assessed by two researchers using the Cochrane Collaboration risk of bias tool. The percentages of high/low/unclear judgements for each criterion across included studies were calculated.

\section{Data analysis/synthesis}

Any observed measure of healthcare worker behaviour was converted into a standardised mean difference (SMD, Cohen's D) comparing intervention and control groups [26]. Odds ratios were converted to SMDs [27]. Where necessary, the sign of the SMD was changed to ensure that a positive SMD represented an improvement in compliance with the desired behaviour.

Where data were from appropriately analysed cluster randomised trials or stepped wedge trials the reported adjusted standard errors were used. Where adjusted standard errors were not reported, we inflated them ourselves to account for clustering [28].

Where data were missing, we searched for companion papers. Missing standard deviations were estimated using any available information (e.g. $p$ values, confidence intervals, range, interquartile range) or by searching for trials with similar outcome measures. For cluster randomised trials, we estimated the intracluster correlation coefficient (ICC) where necessary by taking the average of results from similar studies.

Where studies, including factorial trials, assessed more than one intervention, data were extracted for any comparisons that were relevant to the review, avoiding double-counting by dividing the number of participants in the control arm evenly between comparisons. Where there was more than one control arm, the comparison that was the purest test of a social norms intervention was utilised. Where a study was an appropriately analysed factorial trial the covariate and standard error that best estimated the effect of a social norms intervention was extracted. 
All studies that reported a primary or secondary outcome measure that could be converted into an SMD were included in meta-analyses. The approach to utilising the five social norms BCTs in the analysis was to subtract the control arm BCTs from those in the intervention arm, to identify those BCTs that were the active ingredients being tested in the trial. The BCT feedback on behaviour was present alongside a social norm BCT in 88 of 100 comparisons and so we combined feedback on behaviour with the social norm BCT with which it appeared for the purpose of primary meta-analyses.

Fixed effects meta-analysis [29] and forest plots, stratified by BCT were used to assess the effect of social norms on the clinical behaviour of healthcare workers and patient health outcomes. Sources of variation in the type of social norm, context and mode of delivery were explored using both exploratory subgroup analysis and meta-regression [30]. Network meta-analysis [20] was used to (a) utilise all available data and therefore maximise power by including trials that compared two or more different types of social norms (in addition to those that compared a social norm intervention to a control) and (b) rank the different types of social norms intervention in order of effectiveness. A fixed effects approach to meta-analysis was adopted to yield a summary of the evidence in these trials (i.e. the average effect), rather than an estimate of a common underlying treatment effect. Random effects analyses are also reported.

Pre-planned sensitivity analyses assessed the robustness of the conclusions by excluding studies: at high risk of bias on key domains (allocation concealment, sequence generation, selective outcome reporting, attrition, other biases); with 'mean percentage' $<20 \%$ or $>$ $80 \%$ (due to expected skewed distribution)' with imputed standard deviations; using estimated ICCs; with and without feedback on desired behaviour.

\section{Results}

\section{Study characteristics}

There were 4428 citations screened at the title and abstract stage; 477 full-text papers were screened, of which 116 unique trials met the inclusion criteria. Ten of these trials did not report usable outcome

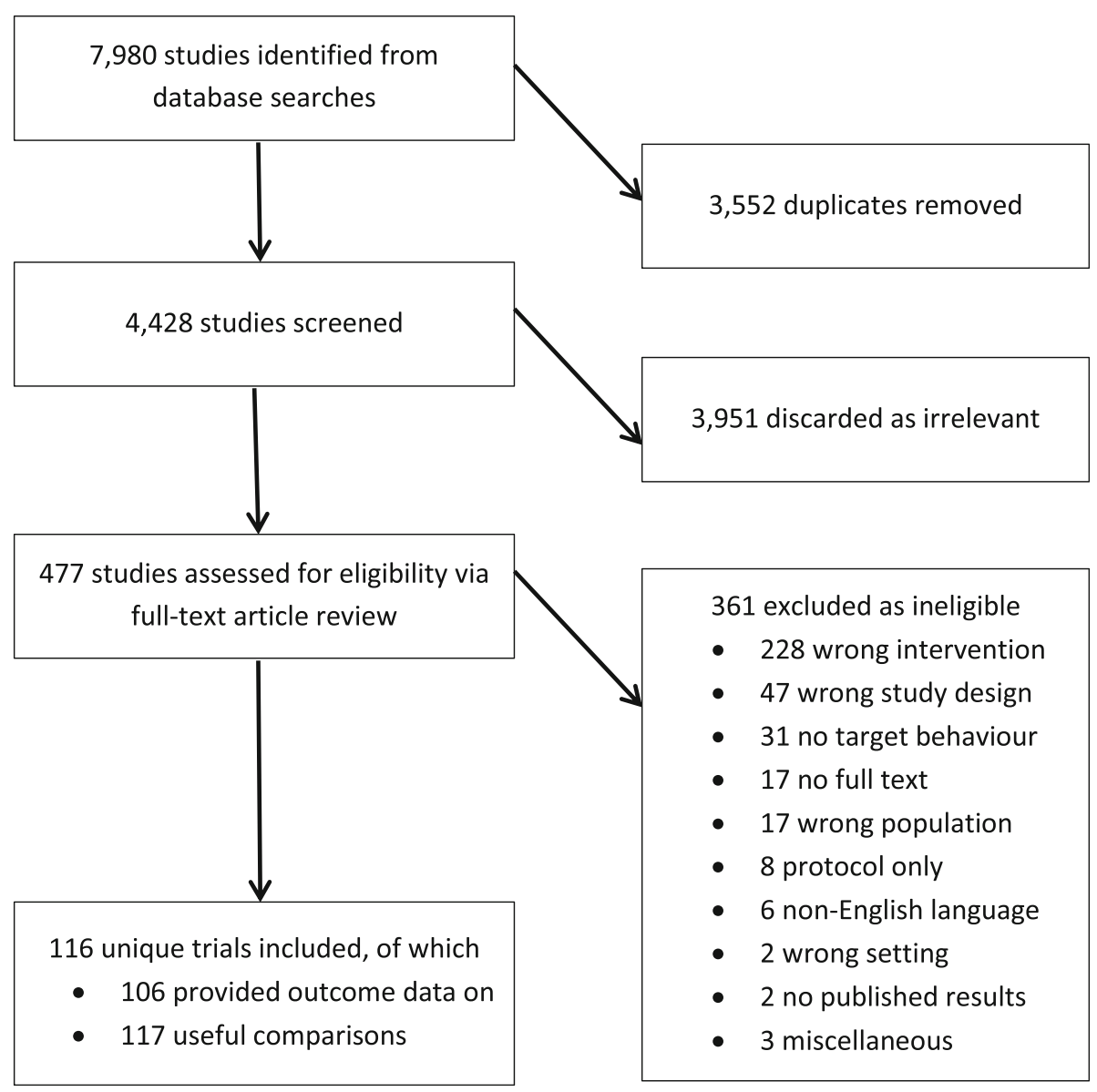

Fig. 1 PRISMA diagram 
data; therefore, a total of 106 trials contributed findings to the review (Fig. 1, Appendix 5). Some studies had more than one trial arm, resulting in 117 included comparisons. The trial and intervention characteristics are summarised in Table 3, and characteristics of each individual comparison are provided in Appendix 6. There were 100 comparisons suitable for meta-analysis. These included studies testing social comparison $(n=79)$ credible source $(n=7)$ and social reward $(n=2)$ against control. Other studies tested more than one social norm together: social comparison and credible source $(n=6)$, social comparison and social reward $(n=2)$, multiple social norms (more than two) together $(n=4)$. Over half of the included trials were conducted in North America; most studies were set in primary care and hospitals, targeting doctors. A broad range of behaviours were targeted including prescribing, management of conditions and test ordering. Two thirds of the trials were cluster RCTs. The interventions were delivered in a variety of formats; a third was delivered on one occasion and the rest on multiple occasions. Most were delivered by someone outside of the target organisation, often an investigator, and three quarters aimed to increase, rather than decrease the behaviour. Some intervention characteristics were poorly reported; format and frequency of delivery were missing in a third of studies (Table 3).

Table 3 Characteristics of included studies

\begin{tabular}{|c|c|c|c|c|c|c|c|c|}
\hline Study characteristic $(n=106)$ & No. & $\%$ & Study characteristic $(n=106)$ & No. & $\%$ & $\begin{array}{l}\text { Intervention characteristic } \\
(n=117)\end{array}$ & No. & $\%$ \\
\hline Country & & & Type of trial & & & Source & & \\
\hline Australia & 8 & 7.5 & Cluster RCT & 69 & 65.1 & Peer & 6 & 5.1 \\
\hline Canada & 15 & 14.2 & Factorial & 4 & 3.8 & Investigators & 83 & 70.9 \\
\hline Denmark & 4 & 3.8 & Randomised controlled trial & 28 & 26.4 & Supervisor or senior colleague & 2 & 1.7 \\
\hline UK & 13 & 12.3 & Stepped wedge & 4 & 3.8 & Patient & 1 & 0.9 \\
\hline Netherlands & 6 & 5.7 & Matched pairs, cluster RCT & 1 & 0.9 & Credible source & 15 & 12.8 \\
\hline USA & 45 & 42.5 & Low baseline performance? ${ }^{\mathrm{a}}$ & & & Other & 1 & 0.9 \\
\hline Other/multiple & 15 & 14.2 & No & 103 & 97.2 & Not reported & 9 & 7.7 \\
\hline Setting & & & Yes & 2 & 1.9 & Internal/external delivery ${ }^{\mathbf{b}}$ & & \\
\hline Primary (GP/GP practice nurses) & 57 & 53.8 & Unclear & 1 & 0.9 & Internal & 17 & 14.5 \\
\hline Hospital (inpatient and outpatient) & 31 & 29.3 & & & & External & 81 & 69.2 \\
\hline Community & 4 & 3.8 & & & & Unclear/not reported & 19 & 16.2 \\
\hline Care/nursing home & 4 & 3.8 & & & & Reference group & & \\
\hline Mixed & 7 & 6.6 & & & & Peer & 97 & 82.9 \\
\hline Other & 3 & 2.8 & Intervention characteristic $(n=117)$ & No. & $\%$ & Professional body & 1 & 0.9 \\
\hline Type of HCP & & & Format & & & Senior person & 9 & 7.7 \\
\hline Doctor (primary care) & 45 & 42.5 & Face-to-face meeting & 16 & 13.7 & Patient(s) & 1 & 0.9 \\
\hline Doctor (secondary) & 19 & 17.9 & Email & 10 & 8.5 & Multiple & 4 & 3.4 \\
\hline Other (nurse/dentist/AHP/pharmacist) & 7 & 6.6 & Written (paper) & 29 & 24.8 & Unclear/not reported & 5 & 4.3 \\
\hline Mixture/whole team & 35 & 33.0 & Separate computerised & 10 & 8.5 & Direction of change & & \\
\hline Target behaviour & & & Mixed & 18 & 15.4 & Increase & 85 & 72.6 \\
\hline Prescribing (incl. vaccinations) & 40 & 37.7 & Unclear/not reported & 34 & 29.1 & Decrease & 30 & 25.6 \\
\hline Handwashing/hygiene & 4 & 3.8 & Frequency & & & Maintenance & 0 & 0.0 \\
\hline Tests/assessments & 21 & 19.8 & Only once & 35 & 29.9 & Unclear & 2 & 1.7 \\
\hline Referrals & 3 & 2.8 & Twice & 10 & 8.5 & Comparator & & \\
\hline Management communications & 25 & 23.6 & More than twice & 45 & 38.5 & Alternative intervention & 15 & 12.8 \\
\hline Other & 2 & 1.9 & Unclear/not reported & 27 & 23.1 & Usual practice & 59 & 50.4 \\
\hline \multirow[t]{2}{*}{ Multiple } & 11 & 10.4 & & & & Attention or waitlist control & 18 & 15.4 \\
\hline & & & & & & Concomitant intervention ${ }^{c}$ & 25 & 21.4 \\
\hline
\end{tabular}

${ }^{\mathrm{a}}$ Does the inclusion criteria target participants based on low target performance?

'The person delivering the intervention internal or external to the target person's organisation?

Intervention that appears in both arms 


\section{Effects of interventions}

\section{Overall effects on clinical behaviours and patient outcomes}

Combined data from fixed effects meta-analysis suggested that social norms interventions were associated with an improvement in healthcare worker clinical behaviour of 0.08 SMDs (95\%CI 0.07 to $0.10, n=100$ comparisons), and an improvement in patient health outcomes of 0.17 SMD (95\%CI 0.14 to 0.20$)$, on average. There was a large amount of heterogeneity with an overall $I^{2}$ value of $85.4 \%$ (primary) and $91.5 \%$ (secondary) suggesting that some studies reported substantially higher or lower effects than the average. However, $I^{2}$ is related to precision and rapidly approaches $100 \%$ when the number of studies is high [31]. Similar conclusions were drawn from random effects meta-analysis an overall improvement in healthcare worker clinical behaviour of 0.16 SMD (95\%CI 0.11 to $\left.0.21, I^{2}=85.4 \%, \tau^{2}=0.043\right)$. Note that the random effects analysis was associated with a larger effect size and wider confidence interval because more weight is given to smaller trials. These results remained robust after all of our pre-planned sensitivity analyses (Appendix 7).

\section{Social norms behaviour change techniques}

Meta-analysis, stratified by social norms BCTs indicated that two of the social norms BCTs had a positive effect on healthcare worker clinical behaviour (Fig. 2): credible source (with or without other BCTs) (SMD 0.30, 95\%CI 0.13 to $0.47, n=7$ ) and social comparison (with or without other BCTs) (SMD 0.06, 95\%CI 0.04 to $0.08, n=$ 77). Social reward may not be effective (SMD 0.03, $95 \%$ CI -0.08 to $0.13, n=2$ ), based on a small sample. We did not find sufficient evidence to examine the effect of the other two social norm BCTs (information about others' approval and social incentive). Multiple social norms delivered together were also effective on average (SMD 0.13, 95\%CI 0.10 to 0.16). When we looked at the most common combinations of social norms BCTs alongside other BCTs, three types of social norms intervention were most effective, on average, compared to control (Table 4): credible source $(0.30,95 \% \mathrm{CI} 0.13$ to $0.47)$; social comparison combined with social reward (0.39, $95 \%$ CI 0.15 to 0.64$)$; and social comparison combined with prompts and cues $(0.33,95 \% \mathrm{CI} 0.22$ to 0.44$)$.

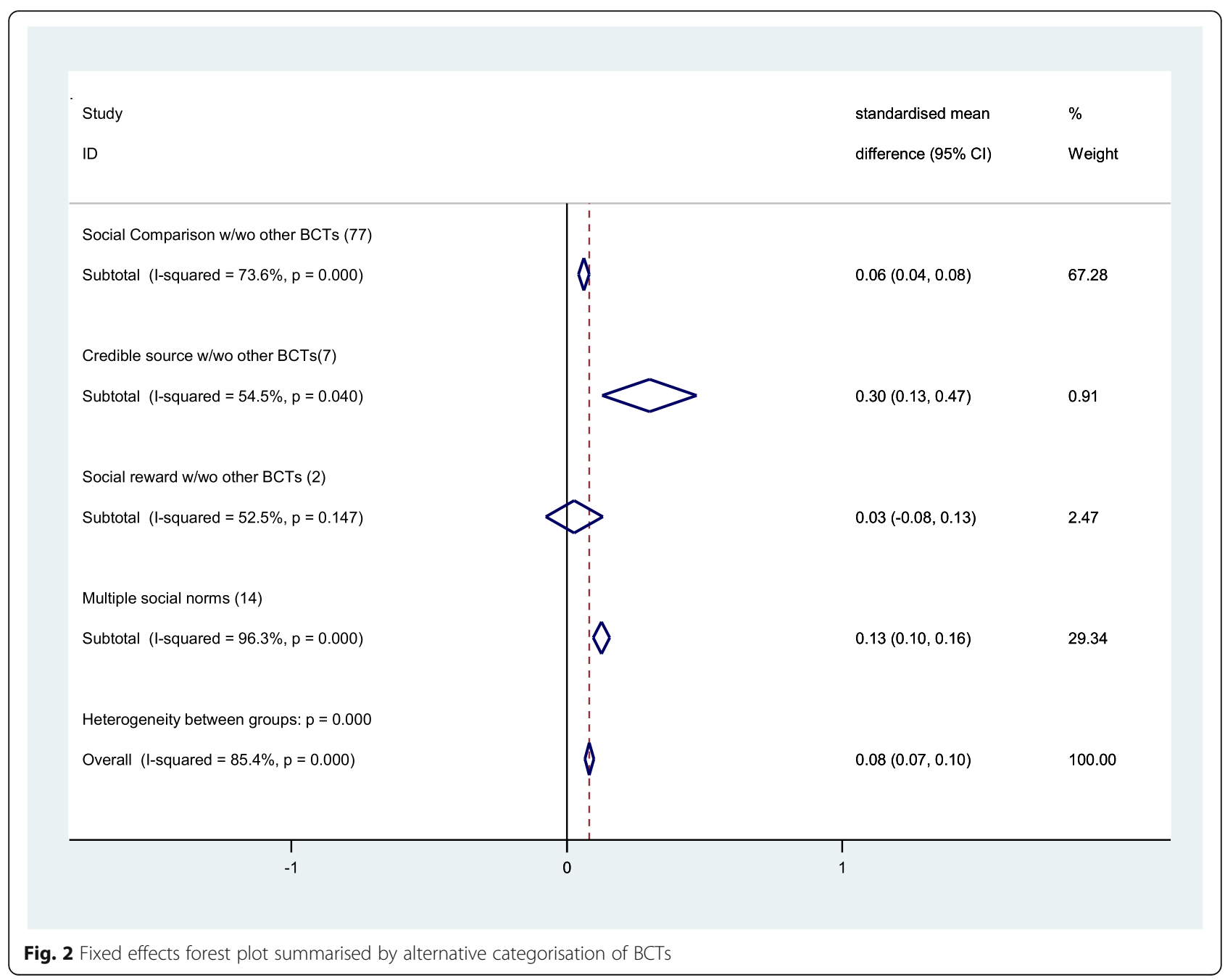


Table 4 Intervention effects calculated from meta-analysis and network meta-analysis, ordered by effect size (intervention $v$ control)

\begin{tabular}{|c|c|c|c|c|}
\hline Type of social norms intervention & $\begin{array}{l}\text { Number of comparisons } \\
\text { for meta-analysis } \\
\text { (network meta-analysis) }\end{array}$ & $\begin{array}{l}\text { SMD meta-analysis } \\
(95 \% \mathrm{Cl}) \\
n=100\end{array}$ & $\begin{array}{l}\text { SMD network meta- } \\
\text { analysis }(95 \% \mathrm{Cl}) \\
n=102\end{array}$ & $\begin{array}{l}\text { Probability of being the } \\
\text { best intervention (\%) }\end{array}$ \\
\hline Social comparison + social reward & 2 & $0.39(0.15$ to 0.64$)$ & 0.39 (0.15 to 0.64$)$ & 59.2 \\
\hline Social comparison + prompts/cues & 5 & $0.33(0.22$ to 0.24$)$ & 0.33 (0.22 to 0.44$)$ & 22.2 \\
\hline Credible source $^{a}$ & 7 & $0.30(0.13$ to 0.47$)$ & 0.30 (0.13 to 0.47$)$ & 18.6 \\
\hline Social comparison + credible source ${ }^{a}$ & $8(10)$ & $0.16(0.12$ to 0.19$)$ & $0.16(0.12$ to 0.20$)$ & 0.0 \\
\hline Social comparison + social support (unspecified) & 7 & $0.10(0.04$ to 0.16$)$ & $0.10(0.04$ to 0.16$)$ & 0.0 \\
\hline Other multiple social norms BCTs & 4 & $0.07(0.03$ to 0.12$)$ & $0.07(0.03$ to 0.12$)$ & 0.0 \\
\hline Social comparison & $33(35)$ & $0.05(0.03$ to 0.08$)$ & $0.05(0.03$ to 0.08$)$ & 0.0 \\
\hline Social comparison + other BCTs & 23 & $0.04(0.00$ to 0.08$)$ & $0.04(0.00$ to 0.08$)$ & 0.0 \\
\hline Social reward & 2 & $0.03(-0.08$ to 0.13$)$ & $0.03(-0.08$ to 0.13$)$ & 0.0 \\
\hline $\begin{array}{l}\text { Social comparison + instructions on how } \\
\text { to perform the behaviour + prompts/cues }\end{array}$ & 5 & $0.01(-0.10$ to 0.11$)$ & $0.01(-0.10$ to 0.11$)$ & 0.0 \\
\hline $\begin{array}{l}\text { Social comparison + info on health } \\
\text { consequences }\end{array}$ & 4 & $\begin{array}{l}-0.14 \\
(-0.33 \text { to } 0.05)\end{array}$ & $-0.14(-0.33$ to 0.05$)$ & 0.0 \\
\hline
\end{tabular}

${ }^{\mathrm{a}}$ With/without other BCTs

Social comparison delivered with credible source $(0.16$, $95 \%$ CI 0.12 to 0.19$)$, on its own $(0.05,95 \%$ CI 0.03 to 0.08 ) or with social support (unspecified) (SMD 0.10, $95 \%$ CI 0.04 to 0.16 ) were all effective, on average, compared to control. This was confirmed by network metaanalysis. Table 5 shows the different contexts and settings for the social norms BCTs and there does not appear to be any obvious patterns of use of the BCTs in particular contexts: social comparison, credible source and social reward are each used in multiple different contexts either alone or alongside other BCTs. Regression analysis suggests that results were consistent even after adjustment for context and setting. Illustrative case studies providing examples of the three intervention types found to be most effective (credible source, social comparison with prompts/cues, social comparison and social reward) are shown in Table 6.

\section{Context and mode of delivery}

Meta-analysis suggested that social norms interventions were effective in a variety of different contexts. The effect was seen with doctors on average (SMD $0.08,95 \% \mathrm{CI} 0.07$ to $0.10, n=68$ ) and other healthcare workers (SMD 0.08, 95\%CI 0.04 to $0.12, n=12$ ), but not with nurses and allied healthcare workers (SMD - 0.01, 95\%CI - .012 to $0.11, n=5$ ). They appeared successful across a range of clinical behaviours, including prescribing (SMD 0.11, 95\%CI 0.09 to $0.13, n=21$ ), arranging, conducting or administering tests/assessments (SMD 0.10, 95\%CI 0.06 to $0.13, n=$ 21 ), and management and communication around health conditions (SMD 0.06, 95\%CI 0.01 to $0.12, n=$ 23), but may be less effective with handwashing
(SMD 0.04, 95\%CI -0.05 to $0.13, n=3$ ) and referrals to other health services (SMD - 0.08, 95\%CI - 0.23 to $0.07, n=3)$. The effects were similar in primary (SMD $0.07,95 \%$ CI 0.05 to $0.09, n=56$ ) and secondary care (SMD $0.12,95 \%$ CI 0.07 to $0.18, n=27$ ) but may be less effective in community (SMD 0.02, $95 \% \mathrm{CI}-0.05$ to $0.10, n=4)$ and care home (SMD $0.03,95 \% \mathrm{CI}-0.05$ to $0.10, n=4)$ settings. The effect appears to be consistent, regardless of whether a peer benchmark $(0.06,95 \% \mathrm{CI} 0.02$ to $.011, n=13)$ or the average $(0.11,95 \%$ CI 0.09 to $0.13, n=67)$ is included. On average, they were slightly less effective in increasing behaviours (e.g. increasing diabetes testing) than at reducing behaviours (e.g. reducing antibiotic prescriptions). The effect was similar regardless of who delivered the intervention and whether it came from within the organisation or from an external source. Interventions that were delivered once $(0.25$, $95 \%$ CI 0.21 to $0.30, n=28$ ) were more effective than those delivered more frequently $(0.06,95 \% \mathrm{CI} 0.04$ to $0.08, n=47)$. Delivery by website was most effective $(0.23,95 \%$ CI 0.15 to $0.31, n=8)$; delivery by email, in writing, and in mixed format were all consistent with the average effect, but face-to-face appeared to be ineffective $(-0.01,95 \% \mathrm{CI}-0.06$ to $0.03, n=14)$. The number of studies in some of these categories was low (nurses and allied healthcare workers, handwashing, referrals to other services, community and care homes), and none of the pre-planned covariates for context and setting appeared to explain much of the heterogeneity in meta-regression, suggesting that any conclusions about context and mode of delivery should remain cautious. 


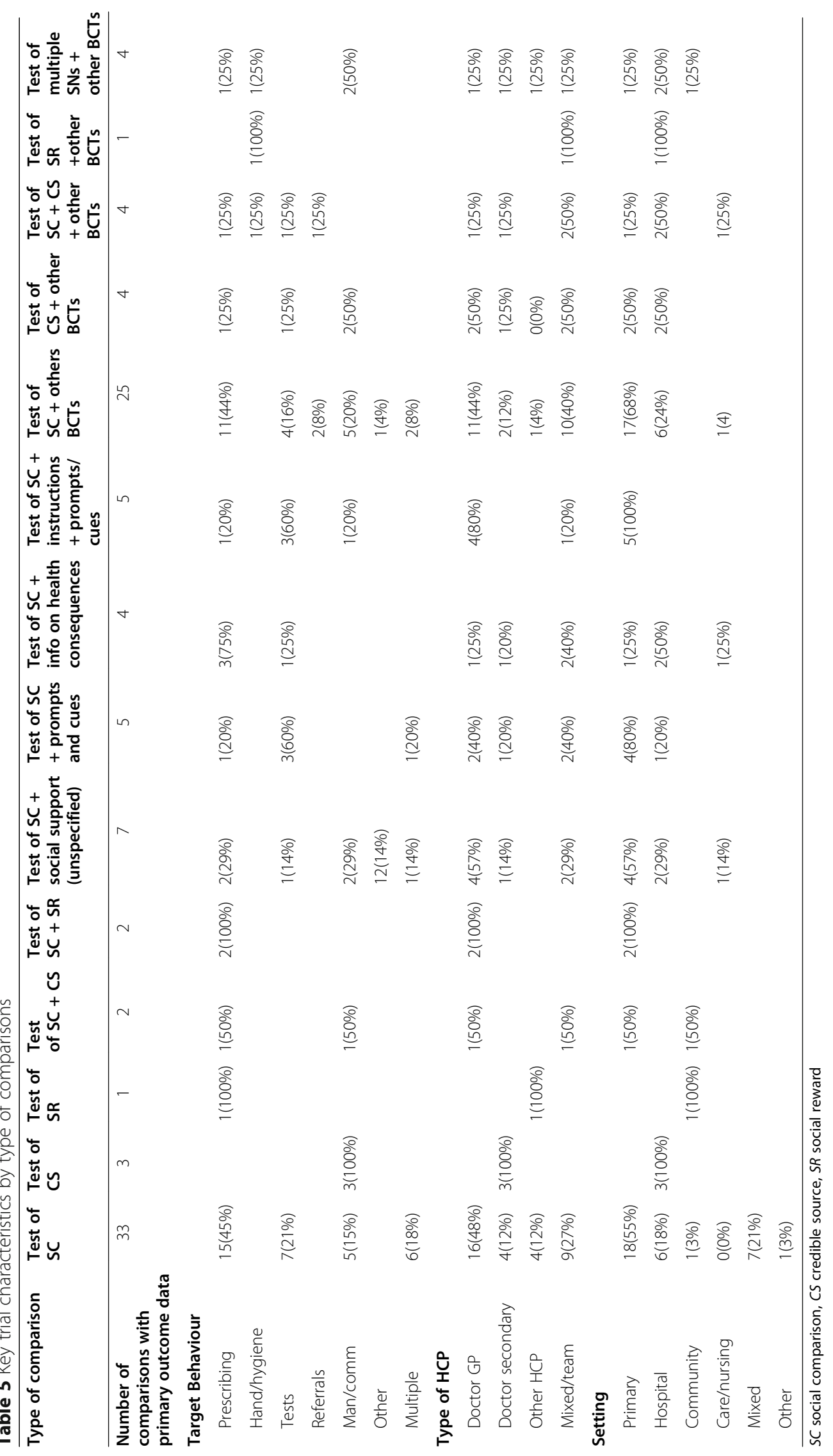



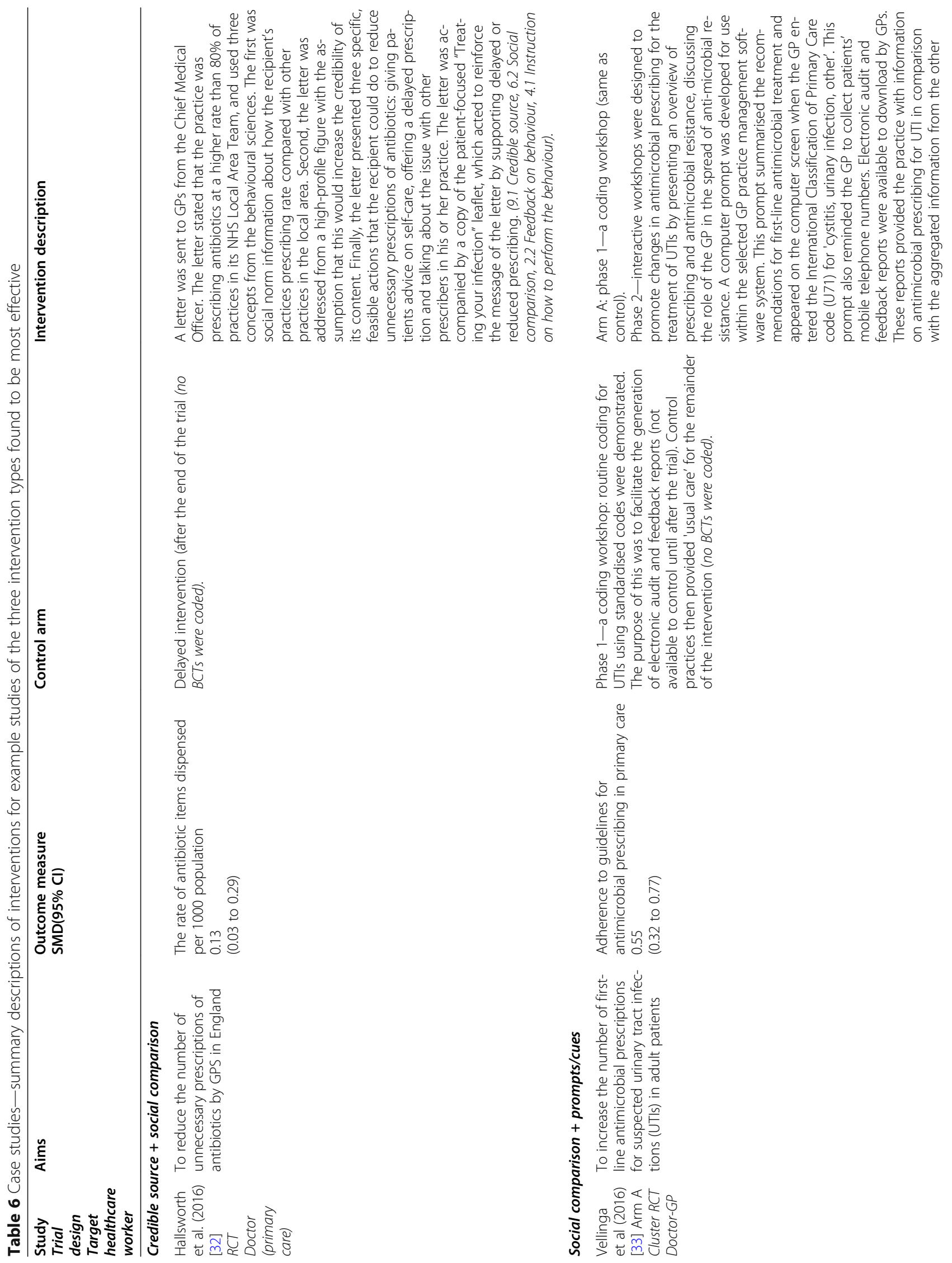


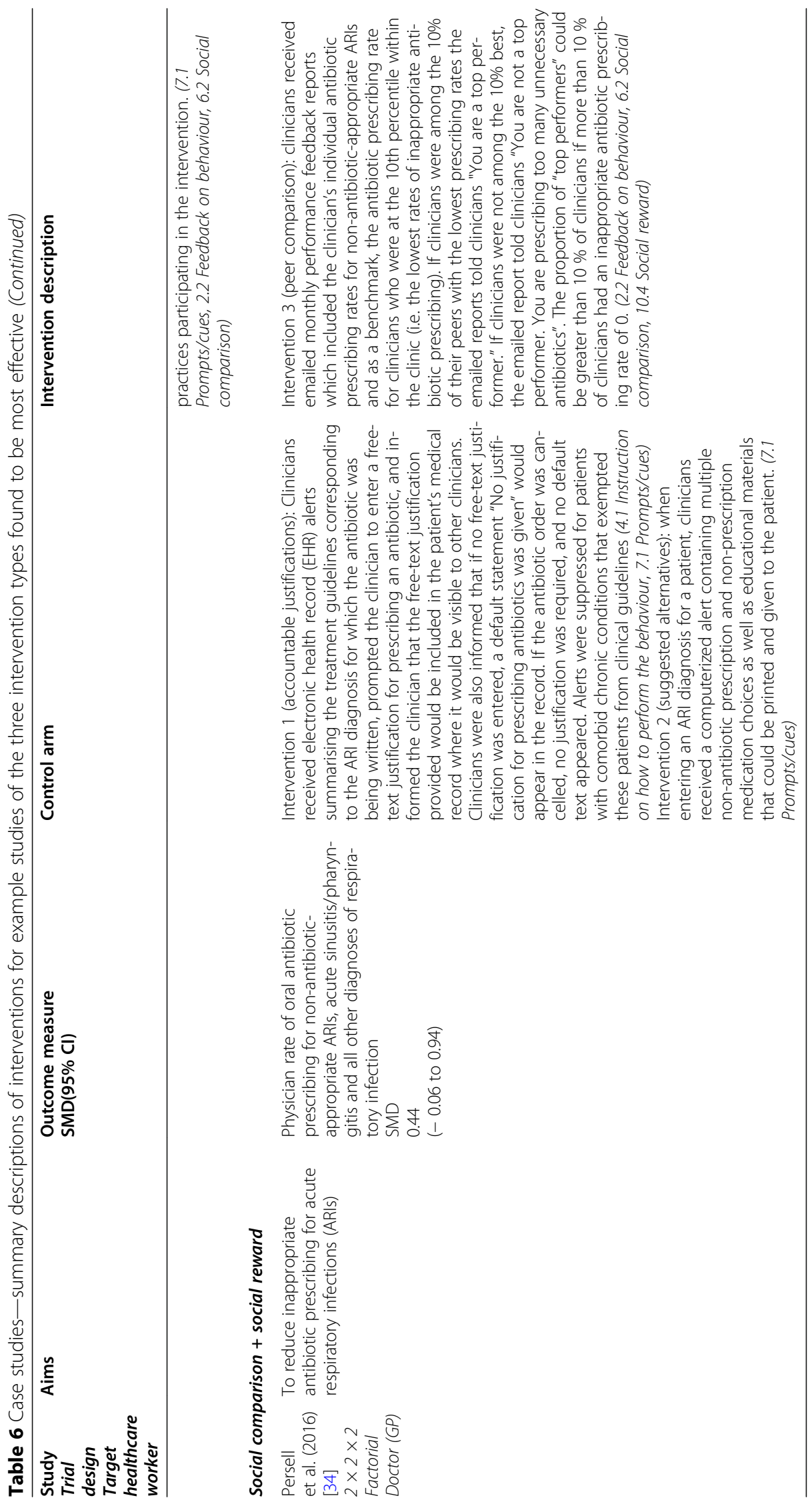




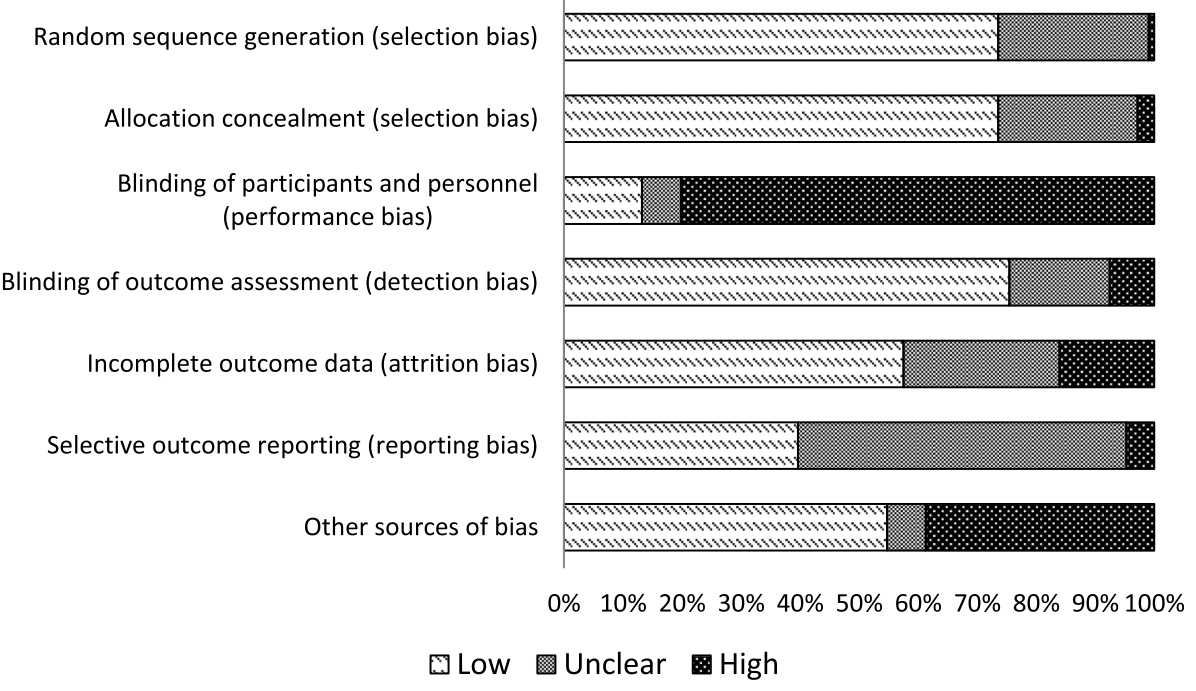

Fig. 3 Review authors' judgements about each risk of bias item (\%)

\section{Risk of bias}

A summary of each risk of bias item across the studies is shown in Fig. 3. Risk of bias was high in $80 \%$ of trials for the blinding of participants and personnel domain and so we cannot rule out the possibility of response bias. This high risk of bias was mainly due to the nature of the interventions (i.e. many of the studies were cluster trials, randomised at the hospital or clinic level, making blinding impractical). In a sensitivity analysis restricting the meta-analysis to trials at low risk of bias for each key domain, the overall treatment effect changed little, suggesting the results were robust. There were five studies at high risk of bias for outcome reporting and 59 with unclear risk of bias. A funnel plot (Fig. 4) identified that the review may be missing some unpublished negative trials, or including more positive trials than expected, suggesting selective outcome reporting.

\section{Discussion}

\section{Summary of evidence}

Social norms interventions can be an effective approach to changing the clinical behaviours of healthcare workers. Meta-analysis showed social norms interventions were associated with an improvement in healthcare worker clinical behaviour outcomes of 0.08 SMDs (95\%CI 0.07 to $0.010, n=100$ comparison) and an

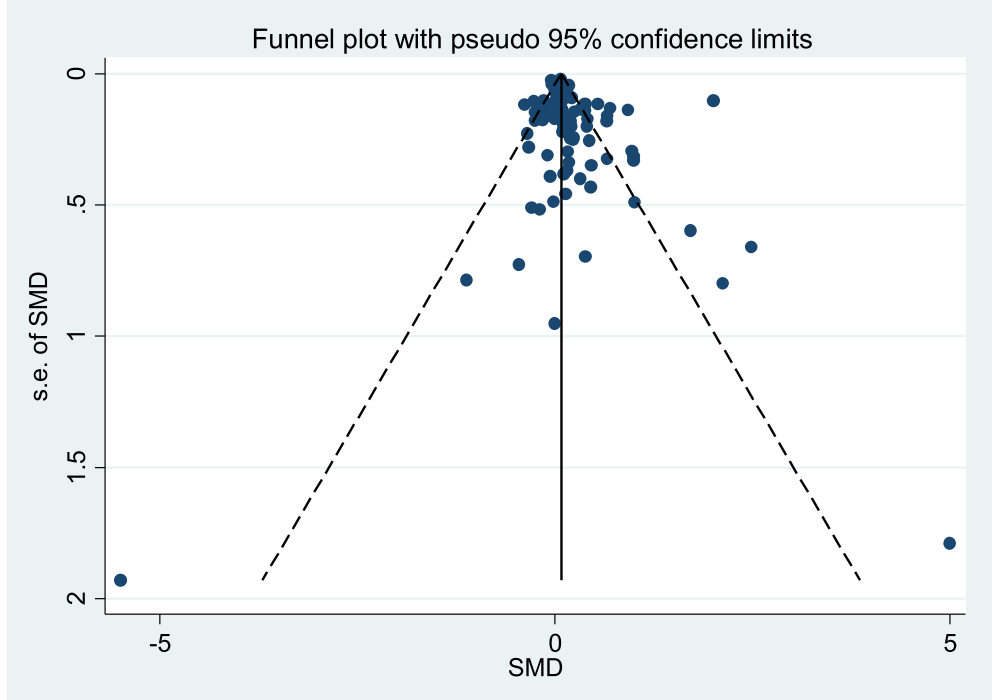

Fig. 4 Funnel plot 
improvement in patient health outcomes of 0.17 SMD ( $95 \% \mathrm{CI} 0.14$ to $0.20, n=14$ comparisons), on average.

There was a large amount of heterogeneity, with some studies reporting substantially higher or lower effects. There was strong evidence from multiple studies that interventions involving social comparison or credible source, with and without other BCTs, were effective on average, both separately and together. Social comparison is effective when combined with various other BCTs including social support (unspecified) but it appears to be most effective when combined with prompts/cues. Social reward appeared not to be effective when used alone but had an above-average effect when combined with social comparison. The effect of social norms interventions remained clear in the meta-regression, even after taking into account context and setting.

Meta-analyses exploring context and delivery showed that social norms were effective with a variety of healthcare workers, in primary and secondary care, and across a range of clinical behaviours. On average, social norms interventions were more effective for reducing than increasing behaviours. Interventions appeared equally effective regardless of whether they came from an internal or external source. In contrast to previous studies [14], delivering the intervention once appeared to be more effective than frequent delivery: one explanation for this, which warrants further investigation, is whether frequent delivery is associated with attempts to change intractable behaviours.

Sensitivity analyses found the overall treatment effect to be robust and not strongly influenced by trials which scored high/unclear risk of bias across key domains. There is a possibility of response bias due to lack of blinding. While it is difficult to blind healthcare workers in these trials, there were examples where the risk of response bias was minimised, e.g. cluster trials where the healthcare worker was not informed of the existence of the trial.

\section{Discussion of findings in relation to the literature}

A Cochrane systematic review $(n=140)$ of the effect of A\&F on healthcare worker behaviour and patient health outcomes [14] found a wide variation in the effect of A\&F and recommended future research to explore how this variation, related to the intervention design, context and recipient [11]. The results of our review contribute to this agenda by suggesting aspects of the design of A\&F interventions that are associated with positive outcomes: (1) highlighting that a credible source approves of the desired behaviour; (2) feedback on an individual's behaviour is likely to be more effective if accompanied by social comparison; (3) complex interventions involving multiple social norms seem to be effective; (4) social comparison seems to be enhanced by the use of prompts and cues, such as computerized pop-ups recommending actions to GPs when particular symptoms or diagnoses are entered into an electronic system [35], but the benefit of prompts and cues may only hold when the healthcare worker understands how to do the behaviour. The effects of social norms were reasonably consistent across a range of healthcare workers, behaviours and settings. In contrast to an earlier review of A\&F [14], delivering the intervention once appeared to be sufficient and sending the intervention by website or other computerised format was most effective. Our results align with findings from a recent synthesis of qualitative literature on $A \& F$ which found that letting healthcare workers know how their performance relates to that of their peers (social comparison) and providing opportunities for peer discussion (social support (unspecified)) were valuable in changing behaviour [6]. However, our finding that face-to-face interventions were less effective than remotely delivered interventions contrasts with results for meta-analyses of smoking cessation interventions where personalised interventions were associated with greater effectiveness [36]. Recent literature suggests that de-implementation is often even more challenging than implementation due to a number of psychological biases: health professionals tend to focus on information that confirms their established beliefs; people are more concerned about losses than gains; and a sense of professional autonomy strengthens attachment to established practices $[37,38]$. Given the challenges of deimplementation our finding that social norms interventions were more effective in increasing behaviour than decreasing it are perhaps not surprising.

A recent overview of 67 systematic reviews on promoting professional behaviour change in healthcare found that the most effective interventions were educational outreach using academic detailing, A\&F and reminders [39]. Using normalization process theory as a theoretical lens, the authors concluded that interventions that seek to 'restructure and reinforce new practice norms' (opinion leaders, educational meetings and materials/guidelines) and those which 'associate practice norms with peer and reference group behaviours' (including A\&F and academic detailing, where a target healthcare worker receives individual support or advice from someone else with expertise in that area) are most likely to be successful in changing clinical behaviour. Combining the two approaches together may be particularly effective, by creating clear rules of conduct and encouraging individuals to follow their peers [39]. Interventions that seek to change attitudes were less likely to be successful. The importance given to peer and reference group behaviours in this previous study justifies our efforts to identify which social norms interventions are associated with success.

The effect sizes seen in this review appear to be similar to other reviews of interventions to change health 
professional behaviour [40]. Baskerville et al found that practice facilitation was associated with an improvement of $0.56 \mathrm{SMD}$ ( $95 \%$ CI 0.43 to 0.68 ) in guideline adoption in primary care. Baker [41] reported that tailored interventions to overcome barriers to change are associated with an odds ratio for the improvement in professional behaviour of 1.51 (95\% CI 1.16 to 2.01) which corresponds to an SMD of approximately 0.24 (95\% CI 0.09 to 0.39 ). The modest effects size seen for social comparison appears in line with that observed by Ivers who found that Audit and feedback improved binary behavioural outcomes by a median of 4.3 percentage points and continuous outcomes by a median of 1.3 percentage points. In a meta-synthesis of systematic reviews of health behaviour change in general, Johnson found effect sizes between 0.08 to 0.45 [42].

\section{Strengths and limitations}

Our search strategy was developed through an iterative process, with input from an Information Scientist. However, it is possible that the strategy may have missed some relevant interventions if social norms BCTs or behaviour change theories were not mentioned in the title or abstract.

We included studies regardless of outcome measure, and we converted any available outcome into a standardized mean difference: this meant we were able to summarise all the available evidence in one analysis. The included trials incorporated a variety of contexts and settings; trial designs and units of analysis. This has led to a heterogeneous review; and we acknowledge the limitations of this approach. The magnitude of effects for the most promising behaviour change interventions were around 0.3 SMDs, which relative to the between study variability $\tau(0.2)$ does seem to indicate an important effect.

Trials were excluded from the review where the intervention did not target a specific behaviour: for example, if the intervention was aimed at a healthcare worker with the intention of reducing patient blood pressure, but did not make explicit what behaviour(s) were expected of the healthcare worker to achieve the reduction. These exclusions occurred because, if a behaviour is not specified, it is not possible to determine whether or not an intervention actually targeted that behaviour and change in that behaviour (our primary outcome) cannot be assessed. This approach is consistent with the coding instructions of the BCTTv1 [16]. There is a potential risk that we have excluded some studies where there was a target behaviour but it was poorly reported.

We used the BCTTv1 [16], which has been based on a significant body of research, to code for BCTs that could be associated with the effectiveness of interventions. However, BCTs were only coded based on published reports and we did not ask study authors for intervention manuals due to time constraints. Therefore, it is possible that our coding did not represent all actual BCTs as designed and delivered. The authors of the BCTTv1 have also acknowledged that extension or modification of the BCTTv1 could be appropriate in the future. It is therefore possible that some BCTs that do not yet feature in the BCTTV1 could have been presented alongside social norms BCTs and were missed during the BCT coding exercise.

Ten small studies without suitable outcome measures were omitted from the meta-analysis and some missing information (such as ICCs and standard deviations) were imputed, but sensitivity analyses suggested no significant impact on the review.

The primary approach to meta-analysis was fixed effects [43], which summarises the evidence in these trials, rather than estimating a common underlying treatment effect [44]. This topic is highly contested, so random effects was also undertaken for the most important analyses, as planned. In all analyses the fixed and random effects approaches produced a result in the same direction, with the random effects approach resulting in a higher effect for the intervention because it gives greater weight to smaller studies. The conclusions of the review would be similar, regardless of whether fixed or random effects were used.

All of the meta-analysis was undertaken on the basis of comparisons: the BCTs in the control arm were subtracted from those in the intervention arm to capture BCTs that were actively tested in each study. The active ingredient was what is left of the intervention when the control arm is taken away. This is a suitable approach to examining the effect of the various social norm BCTs, but a limitation is that some interaction effects may have been missed.

The asymmetry of the funnel plot suggested that the review may have missed some unpublished negative trials or be at risk of bias from selective outcome reporting. The resources were not available for translation or to request unpublished material from authors of included studies, so some relevant studies may have been omitted. A single behaviour outcome was selected from every trial using published reports which may have put the review at risk from selective outcome reporting; priority was given to the pre-specified primary outcome. Sensitivity analysis including only those trials with either a relevant pre-specified primary outcome or single relevant behavioural outcome suggested that results were robust to selective outcome reporting.

\section{Further research}

Credible source has been identified as an effective intervention component. Yet, it is not commonly used in the health setting to change the behaviour of healthcare workers (only $18 \%$ of the comparisons identified in the present review). This may be due to credible source lacking formal conceptualisation in the health setting so, 
whilst it may be used in practice, it is not well-reported. Additional work is needed to develop credible source interventions for use in the NHS, such as, whom the target audience would consider as credible sources: for example, seniority may not necessarily be perceived as the same as credible. As a first step, a narrative synthesis of the trials using credible source in this review, together with the qualitative papers, process evaluations and protocols associated with those trials, would provide further insights into the credible source interventions that are associated with more successful outcomes. Qualitative work with healthcare workers, managers and policymakers is also needed to understand the acceptability and feasibility of credible source, social comparison and social reward interventions and to understand who the most credible sources are.

Social comparison is currently used more frequently with healthcare workers than credible source. We identified a high level of heterogeneity in the effectiveness of social comparison. We have started to unravel this heterogeneity, and this research suggests that social comparison can successfully be enhanced by the addition of social reward, prompts and cues or social support (unspecified); but further research is warranted. The heterogeneity could potentially be explained by differences in how social comparisons are facilitated and what kind of comparisons are made, and not simply by the combination of BCTs it is delivered with or without. For example, social comparisons may have a different effect depending on the reference frame (e.g. whether one identifies with those compared to) or depending on the direction of the comparison (i.e. upward or downward comparison). Further investigation into the factors that moderate the effect of social comparison is warranted.

The methodological quality of trials was mixed. The review included some large factorial trials that tested several behaviour change interventions simultaneously, which can be an efficient design for exploring different components of behaviour change interventions and their interactions. Multiphase Optimization Strategy may be a useful framework that can be applied to factorial designs for identifying which combination and sequence of components (e.g. BCTs and mode of delivery) can produce optimal outcomes [45]. Some trials also used novel methods to minimize bias such as 'attention' controls where participants were given the identical behaviour change intervention for an alternative target behaviour: this type of design is to be encouraged.

\section{Conclusions}

Social norms interventions are an effective method of changing healthcare worker clinical behaviour. Although the overall result is modest and very variable, there is the potential for social norms interventions to be applied at scale and have a significant effect on clinical behaviour and resulting patient health outcomes. Both credible source and social comparison were effective. Social comparison was particularly effective when combined with prompts and cues. These interventions were found to be effective in a variety of NHS contexts and across a range of modes of delivery.

\section{Supplementary Information}

The online version contains supplementary material available at https://doi. org/10.1186/s13012-020-01072-1.

Additional file 1: Appendix 1. Search Strategy. Appendix 2. Data

Extraction Form. Appendix 3. Behaviour Change Techniques (BCTS) Extraction Form. Appendix 4. Inter-Rater Agreement for BCT Coding. Appendix 5. Included Study References. Appendix 6. Study and Intervention Characteristics of Included Comparisons. Appendix 7. Sensitivity Analyses.

\section{Abbreviations}

A\&F: Audit and Feedback; BCT: Behaviour Change Technique; BCTTV1: Behaviour Change Techniques Taxonomy Version 1; BNI: British Nursing Index; Cl: Confidence Interval; CINAHL: Cumulative Index of Nursing and Allied Health Literature; Cochrane CENTRAL: Cochrane Central Register of Controlled Trials; EMBASE: Excerpta Medica dataBASE; GP: General practitioner; ICC: Intra-class correlation coefficient; MEDLINE: Medical Literature Analysis and Retrieval System Online; MeSH: Medical Subject Headings; PABAK: Prevalence-Adjusted and Bias-Adjusted Kappa; PRIS MA: Preferred Reporting items for Systematic Reviews; PROSPERO: The International Prospective Register of Systematic Reviews; RCT: Randomised controlled trial; SMD: Standard mean difference; SN: Social norm

\section{Acknowledgements}

We thank the independent members of the Study Steering Committee: Robbie Foy (Chair), Professor of Primary Care, University of Leeds; Marie Johnston, Professor of Health Psychology, University of Aberdeen, Sofia Dias, Professor in Health Technology Assessment, University of York, and Manoj Mistry, lay member. They were very generous with their time and provided encouragement and wise counsel throughout the project. Marie Johnston came all the way from Aberdeen to run a training workshop on BCT coding, and we are also grateful to her for providing comments on an earlier draft of this manuscript. Thank you to Jane Roberts for writing and conducting the searches and double-coding the BCTs. We are grateful to Jack Wilkinson who was employed as a researcher in the early stages of the review until he was successful in winning a University of Manchester presidential fellowship.

\section{Authors' contributions}

MYT (Research Associate, Health Psychology) wrote the first draft; all authors commented on drafts and approved the manuscript. SC (Senior Lecturer, Health Services Research and Statistics) conceived of the idea for the review and managed the project. SC, RP (Senior Lecturer, Health Psychology), SR, BB (Senior Academic GP and Honorary Consultant) and MYT contributed to the protocol. RP was the lead for health psychology, SR was the lead statistician and BB was the clinical lead. MYT, SC and SR were involved in the screening of studies. MYT, SC, SR and EH (Research Associate, Statistics) did the data extraction. MYT, SC and RP (with Jane Roberts) did the BCT coding. SR (Senior Research Fellow, Statistics) converted all the outcome measures to SMDs, conducted the metaanalysis, meta-regression and network meta-analysis of the healthcare worker behaviour outcomes and prepared the results for publication. EH conducted the meta-analysis of patient health outcomes. MYT, LM (PhD student,

Psychology) and SC helped to prepare the data for analysis and undertook other descriptive analysis. The author(s) read and approved the final manuscript.

\section{Funding}

This project is funded by the National Institute for Health Research (NIHR) Health Services and Delivery Research, reference 17/06/06 - The impact of social norms interventions on clinical behaviour change among healthcare workers: a systematic review. The views expressed are those of the authors and not necessarily those of the NIHR or the Department of Health and Social Care. 


\section{Availability of data and materials}

The datasets used and analysed during the current study are available from the corresponding author on reasonable request.

\section{Ethics approval and consent to participate} Not applicable.

\section{Consent for publication}

Not applicable.

\section{Competing interests}

The authors declare that they have no competing interests.

\section{Author details}

${ }^{1}$ Centre for Biostatistics, Division of Population Health, Health Services Research and Primary Care, School of Health Sciences, Faculty of Biology Medicine and Health, University of Manchester, Oxford Road, Manchester M13 9PL, UK. ${ }^{2}$ National Institute of Health Research Behavioural Science Policy Research Unit, Population Health Sciences, Baddiley-Clark Building, Faculty of Medical Sciences, Newcastle University, Newcastle Upon Tyne NE2 4AX, UK. ${ }^{3}$ Manchester Centre for Health Psychology, Division of Psychology and Mental Health, School of Health Sciences, Faculty of Biology Medicine and Health, University of Manchester, Manchester M13 9PL, UK. ${ }^{4}$ Health e-Research Centre, Farr Institute for Health Informatics Research, Faculty of Biology Medicine and Health, University of Manchester, Manchester M13 9PL, UK. ${ }^{5}$ Centre for Primary Care, School of Health Sciences, Faculty of Biology Medicine and Health, University of Manchester, Manchester M13 9PL, UK.

Received: 12 May 2020 Accepted: 9 December 2020

Published online: 07 January 2021

\section{References}

1. Pirmohamed M, James S, Meakin S, Green C, Scott AK, Walley TJ, et al. Adverse drug reactions as cause of admission to hospital: prospective analysis of 18820 patients. BMJ. 2004;329(7456):15-9.

2. Howard RL, Avery AJ, Slavenburg S, Royal S, Pipe G, Lucassen P, et al. Which drugs cause preventable admissions to hospital? A systematic review. British journal of clinical pharmacology. 2007;63(2):136-47.

3. Courtenay M, Rowbotham S, Lim R, Peters S, Yates K, Chater A. Examining influences on antibiotic prescribing by nurse and pharmacist prescribers: a qualitative study using the Theoretical Domains Framework and COM-B. BMJ Open. 2019;9(6):e029177.

4. Paluck ELB, L. Social norms marketing aimed at gender based violence: a literature review. New York: International Rescue Committee; 2010.

5. Schultz PW, Nolan JM, Cialdini RB, Goldstein NJ, Griskevicius V. The Constructive, Destructive, and Reconstructive Power of Social Norms. Psychological Science. 2007;18(5):429-34.

6. Brown B, Gude WT, Blakeman T, van der Veer SN, Ivers N, Francis JJ, et al. Clinical Performance Feedback Intervention Theory (CP-FIT): a new theory for designing, implementing, and evaluating feedback in health care based on a systematic review and meta-synthesis of qualitative research. Implement Sci. 2019;14(1):40.

7. Festinger L. A Theory of Social Comparison Processes. Human Relations. 1954;7(2):117-40.

8. Tajfel H, Turner JC. The social identity theory of inter-group behavior. In: Worchel S, Austin WG, editor. Psychology of Intergroup Relations. Chicago: Nelson-Hall; 1986.

9. Aizen I. The Theory of Planned Behavior. Organizational Behavior and Human Decision Processes. 1991;50(2):179-211.

10. Cialdini RB, Kallgren CA, Reno RR. A focus theory of normative conduct: a theoretical refinement and reevaluation of the role of norms in human behavior. Advances in Experimental Social Psychology. 1991;24:201-34.

11. Ivers NM, Sales A, Colquhoun $H$, et al. No more 'business as usual' with audit and feedback interventions: towards an agenda for a reinvigorated intervention. Implement Sci. 2014;9:14. https://doi.org/10.1186/1748-5908-9-14.

12. Carney PA, Abraham L, Cook A, Feig SA, Sickles EA, Miglioretti DL, et al. Impact of an educational intervention designed to reduce unnecessary recall during screening mammography. Academic Radiology. 2012;19(9):1114-20.

13. Ivers NM, Tu K, Young J, Francis JJ, Barnsley J, Shah BR, et al. Feedback GAP: pragmatic, cluster-randomized trial of goal setting and action plans to increase the effectiveness of audit and feedback interventions in primary care. Implement Sci. 2013;8:142

14. Ivers N, Jamtvedt G, Flottorp S, Young JM, Odgaard-Jensen J, French SD, et al. Audit and feedback: effects on professional practice and healthcare outcomes. Cochrane Database Syst Rev. 2012;6:CD000259.

15. Gardner B, Whittington C, McAteer J, Eccles MP, Michie S. Using theory to synthesise evidence from behaviour change interventions: the example of audit and feedback. Social science \& medicine. 2010;70(10):1618-25.

16. Michie S, Richardson M, Johnston M, Abraham C, Francis J, Hardeman W, et al. The behavior change technique taxonomy (v1) of 93 hierarchically clustered techniques: building an international consensus for the reporting of behavior change interventions. Ann Behav Med. 2013;46(1):81-95.

17. Cotterill S, Powell R, Rhodes S, Brown B, Roberts J, Tang MY, et al. The impact of social norms interventions on clinical behaviour change among health workers: protocol for a systematic review and meta-analysis. Syst Rev. 2019;8(1):176.

18. Deeks JJ, Higgins JPT, Altman DG. Chapter 10: Analysing data and undertaking meta-analyses. In: Cochrane Handbook for Systematic Reviews of Interventions version 60 (updated July 2019); 2019.

19. Thompson SG, Higgins JPT. How should meta-regression analyses be undertaken and interpreted? Statistics in Medicine. 2020;21:1559-73.

20. Chaimani A, Higgins JPT, Mavridis D, Spyridonos P, Salanti G. Graphical tools for network meta-analysis in STATA. PLoS One. 2013;8(10):e76654.

21. Moher D, Liberati A, Tetzlaff J, Altman DG, Group P. Preferred reporting items for systematic reviews and meta-analyses: the PRISMA statement. PLoS Med. 2009;6(7):e1000097.

22. Covidence. Covidence: better systematic review management 2019 [Available from: www.covidence.org].

23. Effect Practice and Organisation of Care. Data collection form: EPOC resources for review authors. Oslo: Norweigian Knowledge Centre for the Health Services; 2013. [Available from: https://epoc.cochrane.org/resources/ epoc-resources-review-authors].

24. Abraham C, Wood CE, Johnston M, Francis J, Hardeman W, Richardson M, et al. Reliability of Identification of Behavior Change Techniques in Intervention Descriptions. Annals of Behavioral Medicine. 2015;49(6):885-900.

25. Chen G, Faris P, Hemmelgarn B, Walker RL, Quan H. Measuring agreement of administrative data with chart data using prevalence unadjusted and adjusted kappa. BMC Medical Research Methodology. 2009;9:5.

26. Murad MH, Wang Z, Chu H, Lin L. When continuous outcomes are measured using different scales: guide for meta-analysis and interpretation. BMJ. 2019;364:k4817.

27. Chinn S. A simple method for converting an odds ratio to effect size for use in meta-analysis. Statistics in Medicine. 2000;19:3127-31.

28. The Cochrane Collaboration 16.3.6. Approximate analyses of clusterrandomized trials for meta-analysis: inflating standard errors. In: Higgins JPT, Green S, editor. Cochrane Handbook for Systematic Reviews of Interventions (Version 510) 2011.

29. Harris RB, M.; Deeks, J.; Harbord, R.; Altman, D.; Steichen, T.; Sterne, J. METAN: Stata module for fixed and random effects meta-analysis. Statistical Software Components. 2006;\$456798.

30. Harbord RMH, J.P.T. Meta-Regression in Stata. The Stata Journal. 2008;8(4): 493-519.

31. Rucker G, Schwarzer G, Carpenter JR, Schumacher M. Undue reliance on I(2) in assessing heterogeneity may mislead. BMC Med Res Methodol. 2008;8:79.

32. Hallsworth M, Chadborn T, Sallis A, Sanders M, Berry D, Greaves F, et al. Provision of social norm feedback to high prescribers of antibiotics in general practice: a pragmatic national randomised controlled trial. The Lancet. 2016;387(10029):1743-52.

33. Vellinga A, Galvin S, Duane S, Callan A, Bennett K, Cormican M, et al. Intervention to improve the quality of antimicrobial prescribing for urinary tract infection: a cluster randomized trial. Canadian Medical Association Journal. 2016;188(2):108-15.

34. Persell SD, Doctor JN, Friedberg MW, Meeker D, Friesema E, Cooper A, et al. Behavioral interventions to reduce inappropriate antibiotic prescribing: a randomized pilot trial. BMC Infectious Diseases. 2016;16(1):373.

35. Duane S, Callan A, Galvin S, Murphy AW, Domegan C, O'Shea E, Cormican M, Bennett K, O'Donnell M, Vellinga A. Supporting the improvement and management of prescribing for urinary tract infections (SIMPle): protocol for a cluster randomized trial. Trials. 2013;14(441):1-13.

36. Black N, Eisma MC, Viechtbauer W, Johnston M, West R, Hartmann-Boyce J, Michie S, de Bruin M. Variability and effectiveness of comparator group 
interventions in smoking cessation trials: a systematic review and metaanalysis. Addiction. 2020;115(9):1607-17.

37. van Bodegom LD, Davidoff F, Marang-van de Mheen PJ. Implementation and de-implementation: two sides of the same coin? BMJ Quality \& Safety. 2017;2(6):495-501.

38. Ubel PA, Asch DA. Creating value in health by understanding and overcoming resistance to de-innovation. Health Affairs. 2015;34(2):239-44.

39. Johnson MJ, May CR. Promoting professional behaviour change in healthcare: what interventions work, and why? A theory-led overview of systematic reviews. BMJ Open. 2015;5(9):e008592.

40. Baskerville NB, Liddy C, Hogg W. Systematic review and meta-analysis of practice facilitation within prinary care settings. Annals of Family Medicine. 2012;10(1):63-74.

41. Baker R, Camosso-Stefinovic J, Gillies C, Shaw EJ, Cheater F, Flottorp S, Robertson N. Tailored interventions to overcome identified barriers to change: effects on professional practice and health care outcomes. Cochrane Database Syst Rev. 2010;3:CD005470.

42. Johnson BT, Scott-Sheldon LAJ, Carey MP. Meta-Synthesis of Health Behavior Change Meta-Analyses. American Journal of Public Health. 2010; 100(11):2193-8.

43. Poole CG, S. Random-effects meta-analyses are not always conservative. American Journal of Epidemiology. 1999;150(5):169-75.

44. Higgins JPT, Lopez-Lopez JA, Becker BJ, Davies SR, Dawson S, Grimshaw JM, et al. Synthesising quantitative evidence in systematic reviews of complex health interventions. BMJ Glob Health. 2019;4(Suppl 1):e000858.

45. Collins LM, Murphy SA, Strecher V. The multiphase optimization strategy (MOST) and the sequential multiple assignment randomized trial (SMART): new methods for more potent eHealth interventions. Am J Prev Med. 2007; 32(5 Suppl):S112-8.

\section{Publisher's Note}

Springer Nature remains neutral with regard to jurisdictional claims in published maps and institutional affiliations.

Ready to submit your research? Choose BMC and benefit from:

- fast, convenient online submission

- thorough peer review by experienced researchers in your field

- rapid publication on acceptance

- support for research data, including large and complex data types

- gold Open Access which fosters wider collaboration and increased citations

- maximum visibility for your research: over $100 \mathrm{M}$ website views per year

At BMC, research is always in progress.

Learn more biomedcentral.com/submissions 